\title{
Consideraciones estadísticas sobre la cuestión de una selección de centros discriminatorios en el análisis de un ensayo clínico. En respuesta al artículo: "Un método para controlar una tasa alta de respuesta al placebo en una comparación de venlafaxina XR y diacepam en el tratamiento a corto plazo de pacientes con trastorno de ansiedad generalizada"
}

\author{
Bruno Falissard
}

12, av. Paul Vaillant-Couturier, 94804 Villejuif cedex, Francia

\section{INTRODUCCIÓN}

El artículo de Hackett y cols. aprovecha la ocasión de un ensayo no concluyente en el tratamiento del trastorno de ansiedad generalizada (TAG) para ocuparse de una cuestión candente: la heterogeneidad de la respuesta entre los centros incluidos en un ensayo y, con más precisión, la heterogeneidad en la respuesta al placebo. La idea subyacente es como sigue. Considérese un ensayo multicéntrico clásico de tres ramas que compara un medicamento nuevo, uno estándar y un placebo. Algunos centros presentarán inevitablemente una diferencia más pequeña entre la eficacia del placebo y la del medicamento estándar. Esta diferencia más pequeña se puede deber, en particular, al azar, a un efecto elevado de la relación paciente/profesional clínico que probablemente anega el efecto específico del medicamento activo, o a un nivel elevado de ruido en las evaluaciones. En efecto, los centros que no son cuidadosos en la evaluación de los criterios de inclusión o en la puntuación de las variables de eficacia tendrán dificultades para discriminar el placebo del medicamento estándar. Una idea intuitiva es entonces descartar estos centros del análisis de eficacia, al menos en una perspectiva exploratoria.

Los autores han seguido esta estrategia. Han subdividido los centros según su capacidad para detectar una diferencia de dos puntos en la HAM-A entre el placebo y el medicamento estándar y proponen anali- zar exclusivamente los datos procedentes de los centros "buenos" (es decir, los centros discriminatorios, llamados verdaderamente sensibles) al comparar el nuevo medicamento con el placebo. Este último análisis parece llevar a resultados lógicos estadísticamente significativos con un nivel de 0,05 .

Por desgracia, este análisis infla el error de tipo I, y el nivel de 0,05 no se garantiza ya. Se pueden considerar dos situaciones para iluminar este punto.

En la primera situación, un centro "bueno" se define como uno que discrimina el placebo del medicamento estándar con superioridad de este último. Imagínese ahora un ensayo donde el medicamento estándar, el nuevo medicamento y el placebo tengan el mismo nivel de eficacia (esto corresponde con la "hipótesis nula" de los estadísticos). Por azar, no obstante, los centros tendrán niveles diferentes de respuesta en cada tratamiento. Por tanto, habrá todavía centros aparentemente "buenos" y "malos". Los centros "buenos" corresponderán en realidad a aquellos para los cuales, por azar, hay una baja respuesta al placebo, una respuesta alta al medicamento estándar o ambas cosas. Por cierto, si se compara el medicamento nuevo con el placebo en los centros "buenos", no será sorprendente encontrar que el medicamento nuevo es artificialmente superior, ya que la respuesta al placebo es, por diseño y como promedio, particularmente baja en estos centros.

\footnotetext{
Falissard B. Statistical considerations about the question of a selection of discriminating centres in the analysis of clinical trial. In response to the paper: "A method for controlling for a high response rate in a comparison of venlafaxine XR and diazepan in the short-term treatment of patients with generalised anxiety disorder”. Eur Psychiatry 2003; 18: 188-189.
} 
En la segunda situación, se define un centro "bueno" como uno que discrimina el placebo del medicamento estándar con superioridad o bien del medicamento estándar o bien del placebo. El problema presentado más arriba no es pertinente ya, puesto que los centros "buenos" corresponderán a centros con una respuesta al placebo particularmente alta o baja (lo mismo para el medicamento estándar). Sin embargo, si se imagina un ensayo donde el medicamento estándar sea realmente superior al placebo, mientras que el nuevo es comparable al placebo, discriminar centros (los centros "buenos") corresponderá sólo a la situación en que el medicamento estándar es superior al placebo. Por cierto, la respuesta al placebo en estos centros estará sesgada hacia una respuesta baja y no será sorprendente encontrar otra vez que el medicamento nuevo es artificialmente superior.

Según un punto de vista más formal, este sesgo corresponde a un inflado del error de tipo I, que no es igual ya al valor tradicional del $5 \%$.

En conclusión, incluso si el problema de los centros no discriminatorios es un problema real, la solución no está sin duda alguna en el análisis sencillo de los únicos centros discriminatorios, porque las pruebas estadísticas de la hipótesis no son ya interpretables. Se han hecho algunas propuestas para hacer frente a este problema [1]; parecen ser aceptables desde un punto de vista estadístico, pero no son lo bastante poderosas. Por supuesto, el aumento de los procedimientos de control de calidad es una respuesta metodológica sensata, pero hay esperanza todavía de encontrar procedimientos estadísticos apropiados.

\section{BIBLIOGRAFÍA}

1 Falissard B. A solution to the problem of centres with high level of measurement error in multi-centres trials with psychopharmacological treatments. Int J Neuropsychopharmacol 2002; 5(Suppl 1): S197.

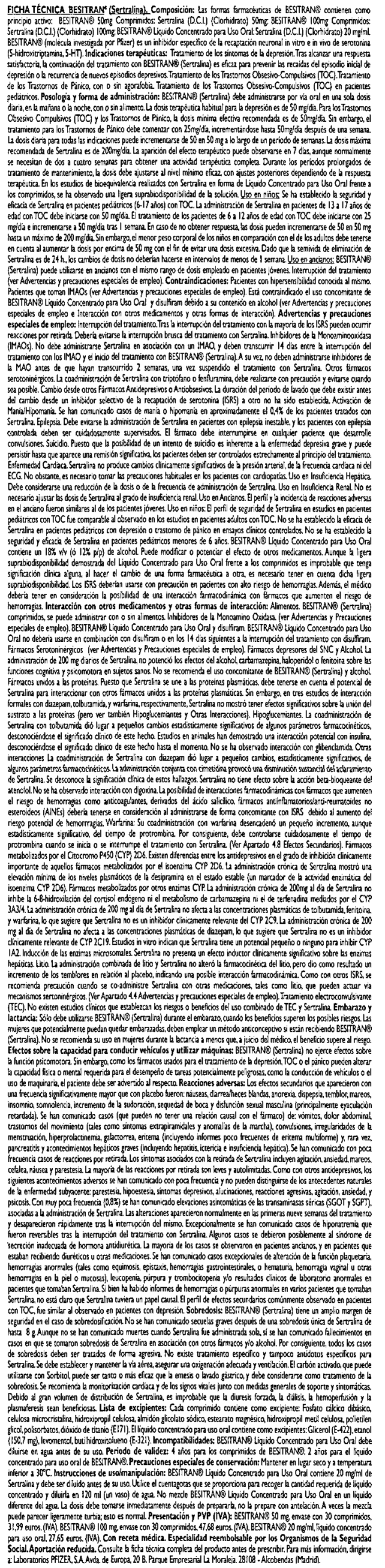



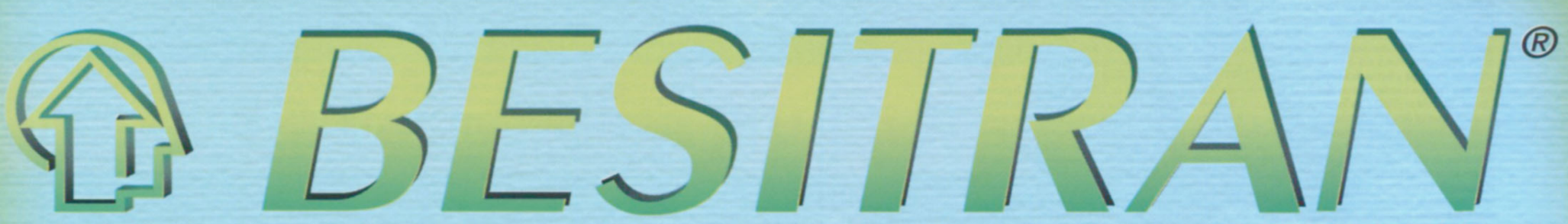

SERTRALINA

$\frac{\text { Ea uilibrio }}{\text { necesario }}$

\section{EFICACIA}

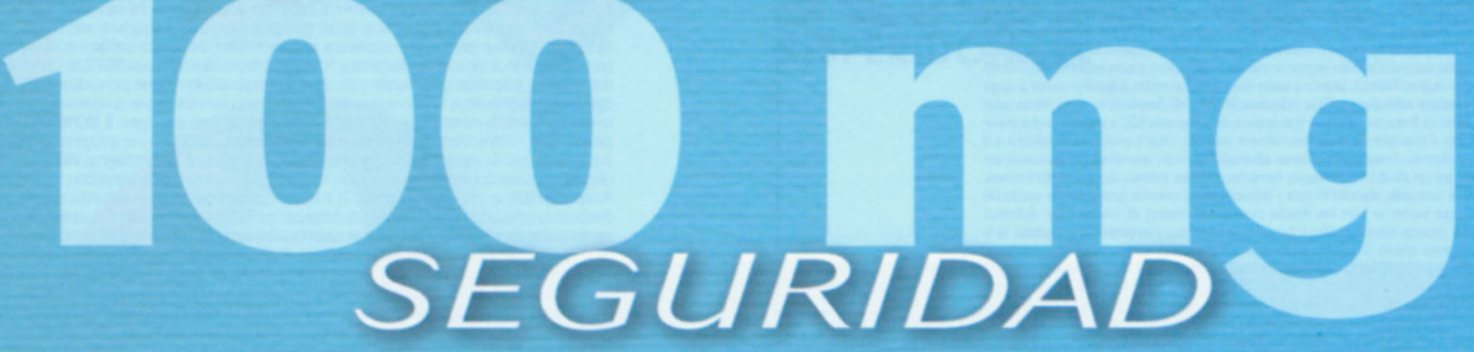

